\title{
A new perspective for phase analysis of radionuclide angiocardiography
}

\author{
Claudine Régis, $M D,{ }^{\mathrm{a}, \mathrm{b}}$ and François Rouzet, $\mathrm{MD}, \mathrm{PhD}^{\mathrm{a}, \mathrm{c}}$ \\ a Nuclear Medicine Department, Hôpital Bichat-Claude Bernard, AP-HP, Paris, France \\ b Department of Medical Imaging, Institut de cardiologie de Montréal, Université de Montréal, \\ Montréal, QC, Canada \\ c Université de Paris and Inserm U1 148, Paris, France
}

Received Jan 5, 2022; accepted Jan 6, 2022

doi: $10.1007 /$ s12350-022-02912-x

\section{See related article, pp. 3086-3098}

Equilibrium radionuclide angiocardiography (RNA) is an imaging modality established since the early 1970s, mainly used for LV function assessment. RNA main advantage is its high accuracy and reproducibility because of the high image contrast. In the last decades, the acquisition procedure has evolved from planar to single photon emission computed tomography (SPECT). SPECT allows to overcome the overlap between adjacent structures by delineating ventricular from atrial chambers with high spatial resolution and abolishing the additional need for background correction. ${ }^{1}$ Left ventricular ejection fraction (LVEF) and right ventricular ejection fraction (RVEF) can be exactly quantitatively derived from the difference in end-diastolic and endsystolic counts of each cavity. In addition, mechanical synchrony can also be precisely assessed on phase analysis. Phase analysis is a map of ventricular contractility in time and can be used to detect both intraand interventricular mechanical dyssynchrony. Even if echocardiography is widely available, inexpensive and non-radiative, RNA is more reproducible, notably to quantify ventricular synchronization. ${ }^{2}$ In contrast to echocardiography, RNA is not operator-dependent, relying on automated contour delineation to determine ventricular volumes, and Fourier phase analysis to

Reprint requests: François Rouzet, MD, PhD, Nuclear Medicine Department, Hôpital Bichat-Claude Bernard, AP-HP, Paris, France; francois.rouzet@aphp.fr

J Nucl Cardiol 2022;29:3099-101.

$1071-3581 / \$ 34.00$

Copyright (C) 2022 The Author(s) under exclusive licence to American Society of Nuclear Cardiology provide contraction delays. RNA is also less limited than echocardiography by patients' morphology, specifically in female and obese patients. Furthermore, RNA is achievable in patients with contra-indications to magnetic resonance imaging such as non-compatible metallic implants and cardiac devices. Myocardial perfusion imaging (MPI) can also assess LV synchrony but compared to RNA, cannot evaluate RV and interventricular dyssynchrony, and has a lower accuracy for LVEF measurement. ${ }^{3}$ Consequently, by providing a straightforward and robust assessment of cardiac function, RNA is considered the gold standard for the accurate determination of LVEF.

In the past years, markers derived from RNA have emerged as prognostic tools. Based on the Frank-Starling principle, LVEF mostly depends on preload, afterload, and contractility, which are reflected in endsystolic volume (afterload and contractility) and enddiastolic volume (preload and contractility). In many cardiac conditions such as heart failure (HF) and ischemic heart disease, LVEF predicts the risk of adverse event. LVEF is also an important factor in evaluating the risk of cardiac surgeries such as coronary artery by-pass graft and valve surgery. Consequently, accurate assessment of LVEF is essential for patients' management and RNA is considered to be the most accurate imaging modality to evaluate LV ejection fraction. ${ }^{4}$ Regarding arrhythmic cardiomyopathies, Le Guludec et al. studied the prognostic value of planar RNA in patients with right ventricular arrhythmias. Patients with any RV abnormalities on RNA had a higher risk of death than those without which had a very low risk of sudden death. RNA RV phase analysis was the only variable able to predict death and the addition of an LV abnormality significantly increased the risk. ${ }^{5}$

Mechanical dyssynchrony is not equivalent to electrical dyssynchrony even if both often concur. 
Indeed, the latter is defined by an asynchronous electrical activation illustrated by a prolonged QRS duration (>120 ms) and studies have shown that mechanical dyssynchrony could exist in more than a third of patients with normal QRS duration. ${ }^{6}$ Mechanical dyssynchrony can be evaluated by time-to-peak strain standard deviation (TPSSD) and left ventricular global longitudinal strain (GLS) on echocardiogram or phase standard deviation (PSD) on RNA. There is some evidence that PSD on RNA could surpass LVEF, TPSSD, and GLS in the evaluation of mechanical dispersion. ${ }^{7}$

In addition, 3-harmonic phase analysis of planar RNA has shown that the arrhythmogenic substrate in Brugada syndrome was associated with a delayed contraction of the RV outflow tract compared to the apex, even in the absence of patent ST-segment modifications on the surface ECG. ${ }^{8}$ In this article, RV contraction heterogeneity assessed by PSD was also associated with the spontaneous ST-segment elevation pattern. In particular, PSD was significantly increased in patients with type 1 pattern, a finding considered to be related to the presence of a markedly slower conduction into the arrhythmogenic substrate in those patients at the time of RNA acquisition.

Other studies found that left intraventricular dyssynchrony on RNA was related to patients' prognosis by independently predicting cardiac events. In a heterogenous population study, Dauphin et al. observed that an interventricular dyssynchrony cut-off value of $25.5^{\circ}$ on RNA was a good predictor of major events defined on a composite criteria including HF progression, heart transplantation, and cardiac death. ${ }^{9}$ Similarly, but in a population of 103 patients with only idiopathic dilated cardiomyopathy, Fauchier et al. found that an increase SD of LV mean phase analysis (SD cut-off value of $50 \mathrm{~ms}$ ) was a strong predictor of cardiac events. After multivariate analysis, SD of LV mean phase and pulmonary capillary wedge pressure were the two independent predictors of cardiac death and worsening of HF leading to heart transplantation. In this study, SD of the RV mean phase analysis and QRS duration correlated also with cardiac events but to a lesser extent.

The objective of cardiac resynchronization therapy (CRT) or biventricular pacing (BiV) is to resynchronize ventricular electromechanical contractility to improve cardiac output ${ }^{10}$ and eventually survival. Selection of patients with HF (New York Heart Association class IIIV) and LVEF $<35 \%$ candidate to BiV is usually limited to QRS duration. Significant reduction in QRS duration and improvements in hemodynamic status during atrial synchronized $\mathrm{BiV}$ is considered to be a good response to $\mathrm{BiV}$. Based on those criteria, $\mathrm{BiV}$ has proven to reduce mortality. ${ }^{6}$ Nonetheless, the need to refine selection criteria has emerged since about $30 \%$ of
HF patients do not improve after CRT. ${ }^{1,6}$ There is cumulating evidence that CRT can be beneficial in patients without prolonged QRS duration ${ }^{11}$ and that demonstration of mechanical dispersion could be more predictive of patient's response. ${ }^{3}$ RNA seems to be an emerging tool to help patient selection for CRT and was shown to be useful in predicting patients' response to CRT. Indeed, in patients with dilated cardiomyopathy and wide QRS, Toussaint et al. found that in the subgroup with LVEF of $>15 \%$ at baseline, interventricular dyssynchrony was predictive of at least a $5 \%$ increase in LVEF at follow-up with a positive predictive value of $83 \%$. $^{2}$ Kerwin et al. also reported improvement in LVEF in patients with interventricular dyssynchrony at baseline who were implanted with BiV. ${ }^{12}$ In another smallscale study, a threshold of $50^{\circ}$ for interventricular contraction heterogeneity on RNA phase analysis correlated with symptoms improvement after LV pacing ${ }^{13}$ and the decrease in the interventricular contraction delay was the most predictive parameter. Interestingly, Tournoux et al showed that CRT might not be beneficial in patients without a certain level of mechanical interventricular dyssynchrony. Indeed, they found no statistical difference in survival between patients with interventricular dyssynchrony $<24^{\circ}$ who were treated with CRT and those without CRT, but a significant survival difference was observed in patient with major dyssynchrony treated with CRT and those without CRT. ${ }^{3}$ Also in this study, dyssynchrony evidenced by RNA was a predictor of poor prognosis among patients with a QRS duration < $150 \mathrm{~ms}$ or non-LBBB pattern and no CRT device, corroborating the independent prognostic value of mechanical dispersion. In brief, RNA seems a promising imaging tool to help patients' selection and monitoring response to CRT.

In this issue of the Journal of Nuclear Cardiology, the study by Beneyto et al. extends the knowledge on the prognostic role of RNA in several aspects. This largescale (937 patients enrolled over 5 years) retrospective monocenter observational study showed that the dispersion of Fourier phases (referred to as phases standard deviation or PSD) derived from gated blood pool SPECT was the main predictor of severe ventricular arrhythmias (VA) occurrence, even after adjustment for LV ejection fraction and strain-derived mechanical dispersion by echocardiography. The results were significant in a wide range of heart diseases, including in ischemic, dilated non-ischemic, and arrhythmogenic right ventricular cardiomyopathies which accounted for two thirds of the study population. Ranging between 50 and 100 per 100,000 , the incidence of sudden cardiac death in the United States and Europe has declined ${ }^{14}$ due to improvement in prevention and treatment of coronary artery disease. However, while poor LVEF is a major 
predictor of severe arrhythmic events, the majority of VA occurs in patient with LVEF $>35 \%$ and thus prediction of VA remains a challenge. ${ }^{7}$ Thus, by being able to predict the occurrence of VA, RNA could be used to identify patient at higher risk. The multivariate analysis including LVEF, GLS, and TPSSD, of the study by Beneyto et al, showed that only PSD on RNA and sex were independently associated with the occurrence of VA. The superiority of RNA over echocardiography in mechanical dispersion assessment could be explained by a poor echogenicity in some patients and by the averaging of strain values over different myocardial layers.

Compared to most previous studies, Beneyto et al. used SPECT instead of planar RNA so that the prognostic information of phase analysis is obtained in addition to left and right ventricular volumes and ejection fraction, asserting the position of RNA among advanced cardiovascular imaging techniques. .

Another novel aspect of the study by Beneyto et al. is the inclusion of a large number of scans acquired on a solid-state detector (CZT) camera. The greater energy resolution, spatial resolution, and contrast of CZT camera allow for better image quality. Also, the sensitivity of such systems enables to reduce both the injected activity to patients and the acquisition duration, facilitating the scheduling of RNA in a busy department. Finally, by confirming the ability of CZT-SPECT RNA to predict VA similarly to conventional NaI cameras, Beneyto et al. gave another perspective for the clinical utilization of CZT cameras. In brief, the increasing availability of CZT-SPECT systems might foster a wider use of RNA, by not only providing accurate measurement of left and right ventricle dimensions and ejection fractions, but also guiding indications for implantable electronic devices and supplying robust prognostic assessment.

\section{Declarations}

\section{Disclosures}

$C R$ is the laureate of the grant "Bourses du Coeur Fondation Familiale Elina et Giuseppe Borselino."

\section{References}

1. Mitra D, Basu S. Equilibrium radionuclide angiocardiography: Its usefulness in current practice and potential future applications. World J Radiol 2012;4:421. https://doi.org/10.4329/wjr.v4.i10. 421 .
2. Toussaint JF, Peix A, Lavergne T, et al. Reproducibility of the ventricular synchronization parameters assessed by multiharmonic phase analysis of radionuclide angiography in the normal heart. Int J Cardiovasc Imaging 2002;18:187-94. https://doi.org/10.1023/a: 1014681314034.

3. Tournoux F, Chequer R, Sroussi M, et al. Value of mechanical dyssynchrony as assessed by radionuclide ventriculography to predict the cardiac resynchronization therapy response. Eur Heart J Cardiovasc Imaging 2016;17:1250-8. https://doi.org/10.1093/eh jci/jev286.

4. Yang Y, Yam Y, Chen L, et al. Assessment of left ventricular ejection fraction using low radiation dose computed tomography. $\mathrm{J}$ Nucl Cardiol 2016;23:414-21. https://doi.org/10.1007/s12350-015 -0123-6.

5. Le Guludec D, Gauthier H, Porcher R, et al. Prognostic value of radionuclide angiography in patients with right ventricular arrhythmias. Circulation 2001;103:1972-6. https://doi.org/10.116 1/01.cir.103.15.1972.

6. Fudim M, Dalgaard F, Fathallah M, et al. Mechanical dyssynchrony: How do we measure it, what it means, and what we can do about it. J Nucl Cardiol 2021;28:2174-84. https://doi.org/10.1007/ s12350-019-01758-0.

7. Kawakami H, Nerlekar N, Haugaa KH, et al. Prediction of ventricular arrhythmias with left ventricular mechanical dispersion: A systematic review and meta-analysis. JACC Cardiovasc Imaging 2020;13:562-72. https://doi.org/10.1016/j.jcmg.2019.03.025.

8. Rouzet F, Algalarrondo V, Burg S, et al. Contraction delay of the $\mathrm{RV}$ outflow tract in patients with Brugada syndrome is dependent on the spontaneous ST-segment elevation pattern. Heart Rhythm 2011;8:1905-12. https://doi.org/10.1016/j.hrthm.2011.07.026.

9. Dauphin R, Nonin E, Bontemps L, et al. Quantification of ventricular resynchronization reserve by radionuclide phase analysis in heart failure patients: A prospective long-term study. Circ Cardiovasc Imaging 2011;4:114-21. https://doi.org/10.1161/CIR CIMAGING.110.950956.

10. Glikson M, Nielsen JC, Kronborg MB, et al. 2021 ESC Guidelines on cardiac pacing and cardiac resynchronization therapy. Eur Heart J 2021;42:3427-520. https://doi.org/10.1093/eurheartj/ehab 364.

11. Morishima I, Sone T, Tsuboi H, et al. Demonstration of left ventricular dyssynchrony and resynchrony by ECG-gated SPECT with cardioGRAF in a patient with advanced heart failure and narrow QRS complex. J Interv Card Electrophysiol 2009;24:1514. https://doi.org/10.1007/s10840-008-9343-x.

12. Kerwin WF, Botvinick EH, O'Connell JW, et al. Ventricular contraction abnormalities in dilated cardiomyopathy: Effect of biventricular pacing to correct interventricular dyssynchrony. J Am Coll Cardiol 2000;35:1221-7. https://doi.org/10.1016/s07351097(00)00555-6.

13. Le Rest C, Couturier O, Turzo A, et al. Use of left ventricular pacing in heart failure: Evaluation by gated blood pool imaging. $\mathrm{J}$ Nucl Cardiol 1999;6:651-6. https://doi.org/10.1016/s1071-3581(9 9)90103-4.

14. Jong-Ming Pang B, Green MS. Epidemiology of ventricular tachyarrhythmia: Any changes in the past decades? Herzschrittmacherther Elektrophysiol 2017;28:143-8. https://doi.org/10. 1007/s00399-017-0503-5.

Publisher's Note Springer Nature remains neutral with regard to jurisdictional claims in published maps and institutional affiliations. 